\title{
Visualization of Kinase Inhibition-Related Adverse Events Using the Japanese Adverse Drug Event Report Database
}

\author{
Takahito Mizuno $^{1,2}$ (D) $\cdot$ Takamasa Sakai $^{3} \cdot$ Kouichi Tanabe $^{3} \cdot$ Takumi Umemura $^{1} \cdot$ Nobuyuki Goto $^{4} \cdot$ Fumiko Ohtsu $^{3}$
}

Accepted: 7 February 2021 / Published online: 9 March 2021

(c) The Author(s) 2021

\begin{abstract}
Introduction Small molecule tyrosine kinase inhibitors (TKIs) inhibit not only the target kinase but also various kinases as off-target inhibitors not mentioned in the package insert. However, there are no reports that comprehensively examine the relationship between adverse events and kinase affinity.

Objective In this study, we combined basic data and clinical data to visualize the relationship between kinase affinity and adverse events, which will be useful for the management of adverse events in clinical practice.

Methods We targeted TKIs that have been used domestically and for which the dissociation constant was obtained as reported by Davis et al. Adverse event data recorded in the Japanese Adverse Drug Event Report (JADER) database provided by the Pharmaceuticals and Medical Devices Agency between April 2004 and January 2018 were used. We calculated the reporting rates of the Standardized MedDRA Queries (SMQ) for the adverse events of interest and visualized the correlation coefficients with kinase affinity. We used the adverse events associated with VEGFR2 and EGFR to assess their validity.

Results We found a correlation among known kinase-related adverse events, suggesting that the methodology may be used as a signal detection method to generate hypotheses for clinical and basic research.

Conclusion Our comprehensive analysis of the kinase affinity of TKIs in this study, which was based on basic TKI kinase affinity data and the clinical data of the reporting rates, suggested that our comprehensive analysis method is useful for generating hypotheses about possible causal relationships between pharmacological effects and adverse events.
\end{abstract}

\section{Introduction}

Advances in the early diagnosis and treatment of cancer have significantly improved the prognosis of cancer patients. In particular, in the field of chemotherapy, advances in the understanding of the molecular biology of cancer are revealing the mechanism of cancer cell growth and metastasis at the molecular level, and based on this knowledge, targeted molecular therapy has become widely used. Of these targeted therapies, small molecule tyrosine kinase inhibitors (TKIs) are administered orally, and contribute to

Takahito Mizuno

g0773259@ccalumni.meijo-u.ac.jp

1 Department of Pharmacy, Tosei General Hospital, 160-Nishioiwake-cho, Seto, Aichi 489-8642, Japan

2 Graduate School of Pharmacy, Meijo University, Aichi, Japan

3 Faculty of Pharmacy, Meijo University, Aichi, Japan

4 Department of Pharmacy, University of Fukui Hospital, Aichi, Japan the patient's quality of life (QOL), for example by reducing hospital visits and invasive procedures and reducing the burden of repeated hospital visits. However, as such TKIs are developed and applied clinically, it has become clear that adverse events with a profile different from that of conventional drugs occur. This is because TKIs act at the signal transduction stage leading to cell proliferation, and affect organs and tissues depending on the signal transduction pathway. Therefore, a causal relationship exists between the kinases inhibited by TKIs and the adverse events that occur. For example, inhibition of vascular endothelial growth factor receptor (VEGFR) 2 is thought to cause hypertension, proteinuria, arterial thromboembolic events, cardiomyopathy, hemorrhage, wound complications, gastrointestinal perforation, and reversible posterior leukoencephalopathy syndrome [1]. Inhibition of epidermal growth factor receptor (EGFR) is known to be related to diarrhea [2]. Therefore, we believe that adverse events can be predicted in advance for drugs that inhibit EGFR and VEGFR2, which is very valuable information for adverse event management in clinical practice. In particular, serious adverse events, which can 


\section{Key Points}

Our findings suggest that this comprehensive method of analyzing kinase affinity and adverse events is useful in finding adverse events that are difficult to notice.

We found that nintedanib-induced hypothyroidism has the potential to be overlooked in Japan, and show that this finding could be used in clinical practice management.

Even if bosutinib- and dasatinib-induced diarrhea is attributable to a combined cause, we showed that it may be due to an off-target kinase affinity.

easily lead to treatment discontinuation, may be monitored to improve patient QOL. However, the relationship between kinase inhibition and adverse events is only a small part of the picture, and many other relationships between kinase inhibition and adverse events are still poorly understood. In clinical practice, the package insert is used to provide information on the kinases targeted by TKIs. TKIs inhibit not only the target kinase but also various kinases as off-target inhibitors not mentioned in the package insert. In contrast, in basic research, the kinase affinity of various TKIs to various kinases has been investigated [3]. However, although there have been reports examining the relationship between certain adverse events and kinase affinity $[4,5]$, there are no reports that comprehensively examine the relationship. In this study, we combined basic data with clinical data to visualize the relationship between kinase affinity and adverse events, which will be useful for generating hypotheses about possible causal relationships between pharmacological effects and adverse events.

\section{Methods}

\subsection{Obtaining Kinase Affinity Data}

TKIs interact with many other off-target kinases in addition to the target kinase. Therefore, we obtained the dissociation constant [Kd (nmol $\left.\left.1^{-1}\right)\right]$ using an affinity map by Davis et al. that screened 442 kinases [3]. We used pKd [ $-\log _{10}$ $\left(\mathrm{Kd} \times 10^{-9}\right)$ ] determined from $\mathrm{Kd}$ as the kinase affinity data, as in Mahé's method [4].

\subsection{Target Drugs}

We included 17 TKIs that have been used domestically and for which the $\mathrm{Kd}$ was obtained as reported by Davis et al. [3].

\subsection{Target Adverse Events}

In Japan, package inserts are divided into two categories: serious side effects and other side effects. Serious adverse events that are likely to lead to discontinuation of treatment are listed as serious side effects. For this reason, our study included all adverse events listed in the serious side effects section of the package inserts of the target TKIs. Since there is no specific Preferred Term (PT) for a kinase and it is expected that various PTs will be reported, Standardized MedDRA Queries (SMQ), a comprehensive search formula, was used. We used the Medical Dictionary for Regulatory Activities/Japanese version (MedDRA/J) Version 21.0. We identified 37 adverse events using SMQ, which are shown in Table 1.

\subsection{Obtaining Adverse Event Data}

Severe adverse events must be avoided if possible in clinical practice. However, high-severity adverse events are often infrequent, limiting the ability to investigate adverse event frequency in a single center. The JADER database, provided by the Pharmaceuticals and Medical Devices Agency (PMDA), is a database of spontaneous reports, and relatively serious adverse events such as post-marketing adverse events that are not captured in clinical trials have been reported there. Since the JADER database contains only cases in which some adverse events occurred, it is not possible to calculate the original incidence rate. Although some studies use the reporting odds ratio (ROR) as the incidence rate of adverse events [4, 6], the ROR is adjusted for the reporting odds of other drugs to perform qualitative evaluations, such as signal detection [7].

We therefore calculated the reporting rates by dividing the number of adverse events reported for each TKI by the total number of reports for each TKI (Reporting rate of drug $A=$ Number of adverse events reported for drug $A$ /Number of all adverse events reports for $\operatorname{drug} A$ ), because there was no need to correct for other drugs. The JADER database was obtained from the PMDA website (http://www.pmda.go.jp). We assumed the frequency of occurrence in clinical practice to be the reporting rates calculated using data reported to the PMDA from April 2004 to January 2018. The format of the JADER database complies with the International Council for Harmonization of Technical Requirements for Pharmaceuticals for Human Use and consists of four tables: patient 
Table 1 SMQ as a target adverse event

\begin{tabular}{|c|c|}
\hline $\mathrm{SOC}$ & SMQ \\
\hline Blood and lymphatic system disorders & Hematopoietic erythropenia, Hematopoietic leukopenia, Hematopoietic thrombocytopenia \\
\hline Cardiac disorders & $\begin{array}{l}\text { Cardiac arrhythmias, Cardiac failure, Ischemic heart disease, Torsade de pointes/QT prolonga- } \\
\text { tion }\end{array}$ \\
\hline Endocrine disorders & Hyperthyroidism, Hypothyroidism \\
\hline Eye disorders & Corneal disorders, Retinal disorders \\
\hline Gastrointestinal disorders & $\begin{array}{l}\text { Acute pancreatitis, Gastrointestinal hemorrhage, Gastrointestinal perforation, Gastrointestinal } \\
\text { ulceration, Noninfectious diarrhea }\end{array}$ \\
\hline Hepatobiliary disorders & $\begin{array}{l}\text { Cholestasis and jaundice of hepatic origin, Hepatic failure, fibrosis and cirrhosis and other } \\
\text { liver damage-related conditions }\end{array}$ \\
\hline Immune system disorders & Anaphylactic reaction \\
\hline Infections and infestations & Infective pneumonia, Toxic septic shock conditions \\
\hline Metabolism and nutrition disorders & $\begin{array}{l}\text { Dehydration, Hyperglycemia/new onset diabetes mellitus, Hyponatremia/SIADH, Tumour } \\
\text { lysis syndrome }\end{array}$ \\
\hline Musculoskeletal and connective tissue disorders & Rhabdomyolysis/myopathy \\
\hline Nervous system disorders & $\begin{array}{l}\text { Hemorrhagic central nervous system vascular conditions, Noninfectious encephalopathy/ } \\
\text { delirium }\end{array}$ \\
\hline Renal and urinary disorders & Acute renal failure, Proteinuria \\
\hline Respiratory, thoracic and mediastinal disorders & Interstitial lung disease, Pulmonary hypertension \\
\hline Skin and subcutaneous tissue disorders & Severe cutaneous adverse reactions \\
\hline Vascular disorders & $\begin{array}{l}\text { Embolic and thrombotic events, arterial, Embolic and thrombotic events, venous, Hemorrhage } \\
\text { terms (excl laboratory terms), Hypertension }\end{array}$ \\
\hline
\end{tabular}

SOC System Organ Class, SMQ Standardised MedDRA Queries

demographic information (demo); drug information (drug); adverse events (reac); and diseases (hist). In this study, drug records where the suspect drug was involved were included in the analysis.

\subsection{Kinase Adverse Event Correlation Map}

We matched the basic data of TKIs and pKd, which is an index of kinase affinity, with the clinical data of TKIs and reporting rate, and determined the correlation between $\mathrm{pKd}$ and reporting rate. We calculated Pearson's correlation coefficient $(r)$ to see the association between the basic data (kinase affinity) and the clinical data (the reporting rates). We defined the following values as: $0.0 \leq|r| \leq 0.2$ : almost no correlation, $0.2<|r| \leq 0.4$ : weak correlation, $0.4<|r| \leq 0.7$ : relatively strong correlation, $0.7<|r| \leq 1.0$ : strong correlation. Background color was added when correlation coefficients above a relatively strong correlation were found. The aforementioned data were used to create the kinase adverse event correlation map. For the kinases that we considered required a detailed analysis of the correlations, we created a scatterplot of adverse events and kinase affinity in TKIs as the reporting rates on the vertical axis and pKd on the horizontal axis to confirm the linear relationship.

\subsection{Verification Method}

For verification, we focused on VEGFR2 and EGFR where many of the relationships between adverse events and kinases have been clearly identified among the 442 kinases. VEGFR2 has been associated with hypertension, proteinuria, arterial thromboembolic events, cardiomyopathy, hemorrhage, wound complications, gastrointestinal perforation, and reversible posterior leukoencephalopathy syndrome, but not with hand-foot syndrome, mucositis, skin reactions, hypophosphatemia, or lipase increase [1, 8-13]. The relationship between VEGFR2 and hypothyroidism and myelosuppression is unclear [1]. EGFR has been associated with diarrhea [2]. Therefore, we validated our methodology by testing whether there were correlations with $\mathrm{pKd}$ with the following adverse events: hypertension, proteinuria, embolic and thrombotic events, arterial, hemorrhage terms (excluding laboratory terms), hemorrhagic central nervous system vascular conditions, gastrointestinal perforation, severe cutaneous adverse reactions, and noninfectious diarrhea in the presence of SMQ. For sensitivity analysis 1, we used the ROR instead of reported proportions to identify correlates of hypertension, proteinuria, embolic and thrombotic events, arterial, hemorrhage terms (excluding laboratory terms), hemorrhagic central nervous system vascular conditions, gastrointestinal perforation, severe cutaneous adverse reactions, and noninfectious diarrhea. For sensitivity analysis 2 , 
we used the kinase-compound activity dataset of Anastassiadis T et al. [14] instead of pKd to identify correlates of hypertension, proteinuria, embolic and thrombotic events, arterial, hemorrhage terms (excluding laboratory terms), hemorrhagic central nervous system vascular conditions, gastrointestinal perforation, severe cutaneous adverse reactions, and noninfectious diarrhea. The kinase-compound activity dataset was calculated by subtracting the kinase activity from $100 \%$.

\subsection{Analyses}

Statistical analysis was performed using SPSS v23 (IBM Japan, Tokyo) and assuming that $r>0.4$ was correlated. In order to give consideration to multiple comparisons of correlations, the correlations subjected to verification were adjusted using the FDR method to minimize the risk of alpha error due to multiple comparisons.

\section{Results}

The total number of registrations with the JADER database during the analysis period was 504,407. Table 2 shows the targeted TKIs and the reporting rates. The number of reports on vandetanib was low because it has only recently been made available on the market compared to other TKIs and because it was only indicated for the treatment of medullary thyroid cancer. The results of the correlation analysis of Pearson's correlation between $\mathrm{pKd}$ and the reporting rates are shown in the Online Supplementary Material (OSM). We categorized them according to kinase family and presented the correlation coefficients (kinase adverse event correlation map). When correlation coefficients higher than a relatively strong correlation were observed in the kinase adverse event correlation map, highlighting was applied to visualize the strong association between kinase affinity and adverse events. The VEGFR2, EGFR, and EGFR families we validated are shown at the top of the kinase adverse event correlation map. The other kinases are shown in alphabetical order. As a result, the correlated adverse events for each kinase family were visualized, and the strength of the association could be visually confirmed. For VEGFR2, a correlation was found with hypothyroidism, for which the association was not clear in basic research. For EGFR, a correlation with non-infectious diarrhea was also observed in the entire EGFR family. For the VEGFR2 we validated, we found significant correlations for hypertension, proteinuria, and gastrointestinal perforation that were clearly related (Table 3). For the EGFR we validated, we found significant correlations to noninfectious diarrhea that were clearly related (Table 3 ). The same analysis was performed using the ROR instead of the reporting rate as a sensitivity analysis, and similar results were obtained, although no significant difference was observed for non-infectious diarrhea. The same results were obtained using the kinase-compound activity dataset instead of $\mathrm{pKd}$, although the correlation coefficient became weaker due to a reduction in the number of kinases and TKIs (Table 4). Figure 1 shows a scatter plot of VEGFR2 affinity and the reporting rates of hypothyroidism. The kinase affinity of axitinib, nintedanib, pazopanib, sorafenib, and sunitinib for VEGFR2 may contribute to the development of hypothyroidism.

Figure 2 shows a scatter plot of EGFR affinity and the reporting rates of non-infectious diarrhea. This suggested that inhibition of EGFR may related to the development of non-infectious diarrhea not only with afatinib, erlotinib, and gefitinib (EGFR-TKIs), which target EGFR, but also with bosutinib, dasatinib, lapatinib, and vandetanib, which offtarget EGFR.

\section{Discussion}

Since TKIs play a central role in cell-signaling pathways, a causal relationship exists between TKIs and the occurrence of adverse events in organs and tissues where the kinases are expressed. However, many TKIs interact with many other families of kinases besides the target kinase [3], making it difficult to understand their relationship to adverse events. Although it is very important for us to know the relationship between the kinase affinity of TKIs and adverse events for the management of adverse events in clinical practice, there are only a few reports that have comprehensively investigated the relationship between kinase affinity and adverse events.

We investigated the correlation between kinase affinity and adverse events of TKIs using the JADER database, which is a database of spontaneous adverse event reports in Japan. The kinase adverse event correlation map developed in this study allowed us to comprehensively visualize the relationship between kinase affinity and adverse events.

In the validation of this study focusing on VEGFR2 and EGFR, a correlation was observed for most known related adverse events, but not for unrelated adverse events, suggesting that the kinase adverse event correlation map may be used to find signals to generate hypotheses for clinical and basic research.

No correlations were found for VEGFR-related adverse events with respect to embolic and thrombotic events, arterial, or hemorrhage terms (excluding laboratory terms). The mechanism by which thromboembolism occurs has been speculated to be the inhibition of NO production by VEGF signaling inhibition, promotion of endothelial cell apoptosis, and platelet activation through the inhibition of regeneration of damaged endothelial cells [10]. However, 


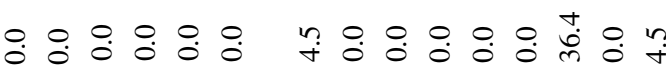

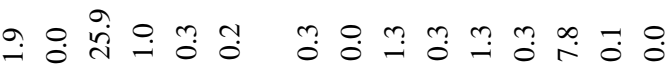
ฟ

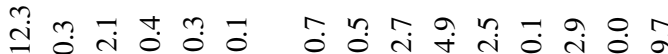

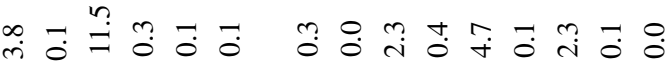
进 元

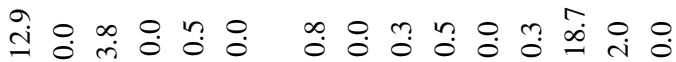

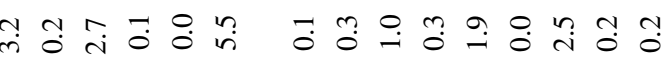

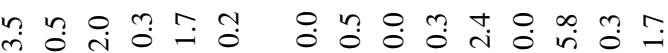
लై

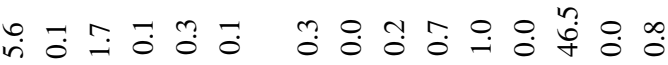

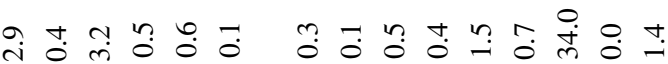

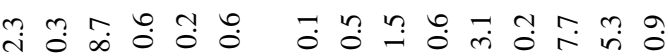

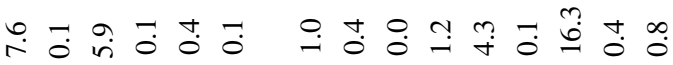

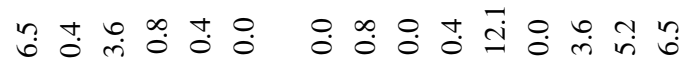
ఇ

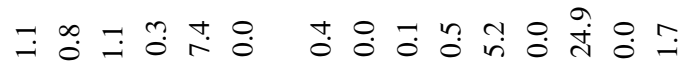

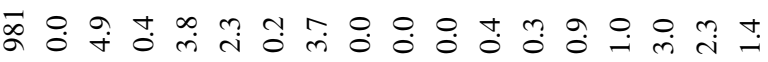

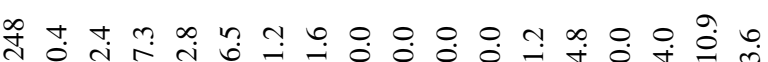

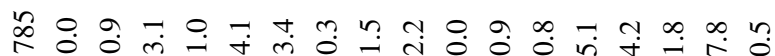

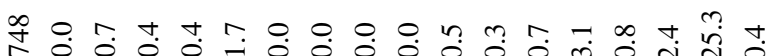
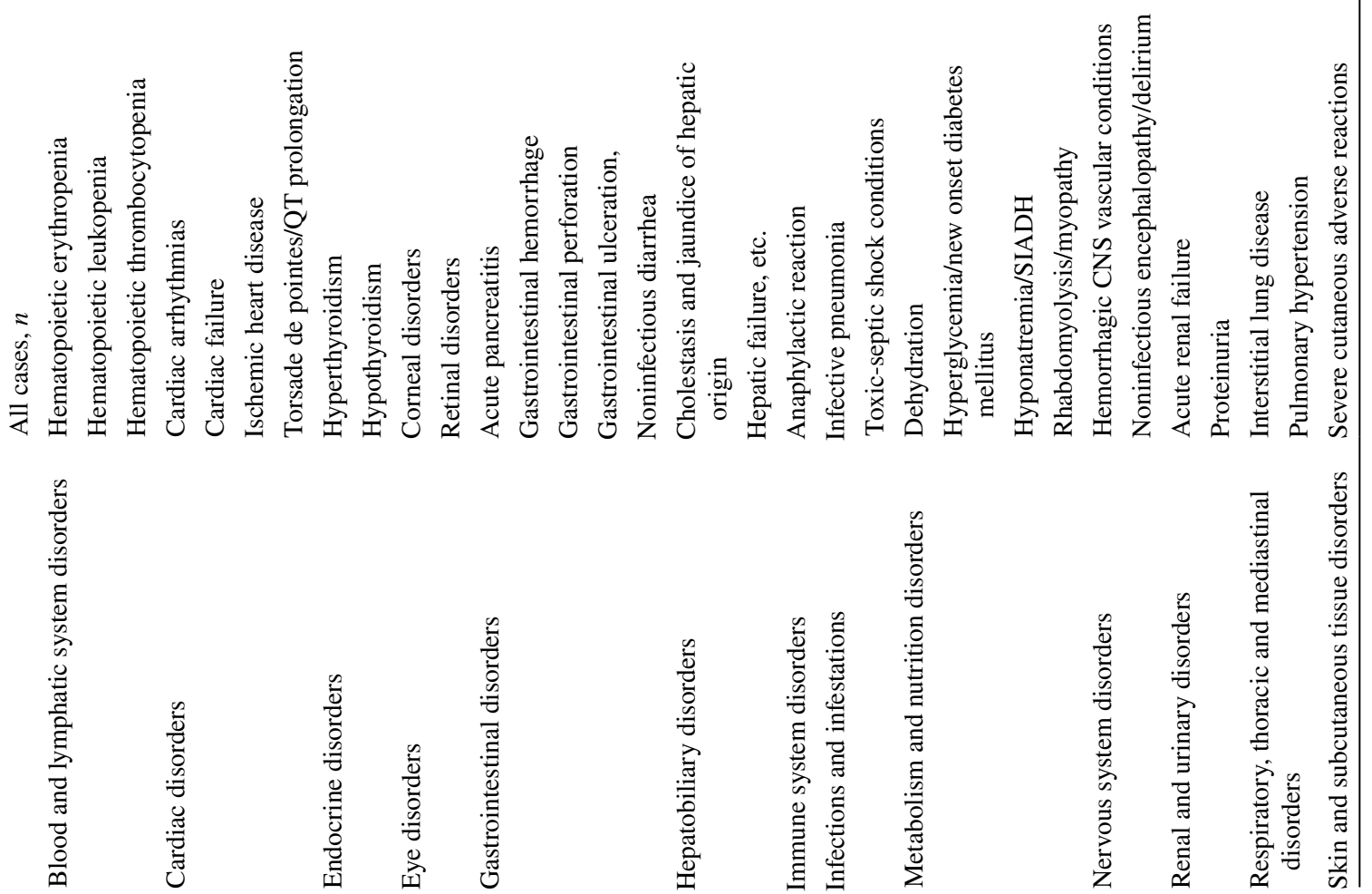


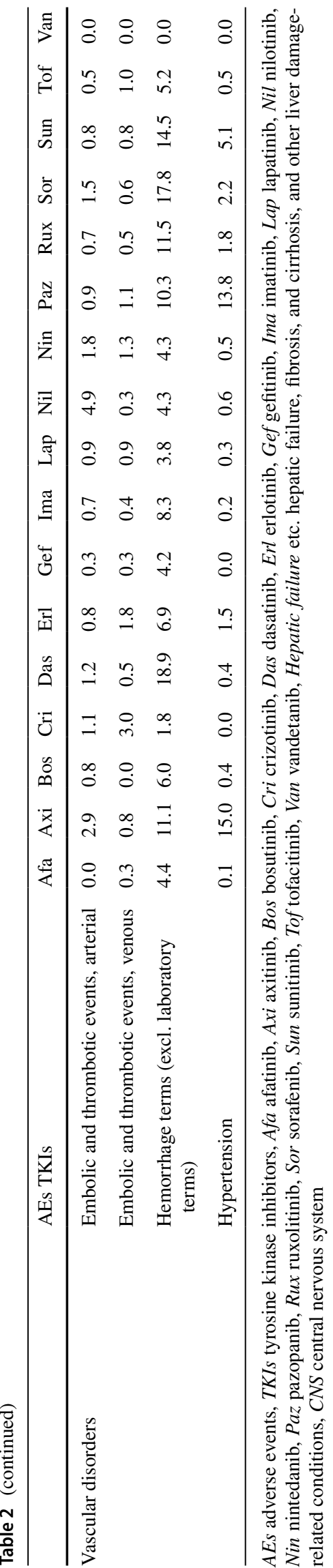

Table 3 Results of calculating Pearson's correlation coefficients between reporting rates of and epidermal growth factor receptor (EGFR) related adverse events and product of dissociation constants of vascular endothelial growth factor receptor (VEGFR) 2 and EGFR

\begin{tabular}{lccl}
\hline SMQ & $r$ & $p$ & $\mathrm{SL}^{\mathrm{a}}$ \\
\hline Hypertension & $\mathbf{0 . 6 5}^{*}$ & 0.0047 & 0.0125 \\
Proteinuria & $\mathbf{0 . 5 8}^{*}$ & 0.0142 & 0.0188 \\
Embolic and thrombotic events, arterial & 0.19 & 0.4684 & 0.0438 \\
Hemorrhage terms (excl laboratory terms) & 0.39 & 0.1168 & 0.0375 \\
Hemorrhagic CNS vascular conditions & $\mathbf{0 . 5 3}$ & 0.0289 & 0.0250 \\
Gastrointestinal perforation & $\mathbf{0 . 7 5}^{*}$ & 0.0005 & 0.0063 \\
Severe cutaneous adverse reactions & $<0.01$ & 0.9944 & 0.0500 \\
Noninfectious diarrhea & $\mathbf{0 . 5 2}^{*}$ & 0.0309 & 0.0312 \\
\hline
\end{tabular}

$S M Q$ Standardised MedDRA Queries, CNS central nervous system $r>0.4$ : bolded and italicized

*Significant difference

${ }^{\text {a }}$ Significance level by false discovery rate

thrombotic events also occur with second-generation TKIs used to treat chronic myeloid leukemia (CML), which have little or no affinity for VEGFR2. The mechanism of thrombotic events is thought to be the inhibition of a number of off-targets in cells involved in thromboembolism, such as vascular endothelial cells, platelets, macrophages, and mast cells [15]. The mechanism of thrombotic events caused by TKIs is complex and cannot be explained by the affinity of a single kinase. Other risk factors, such as older age and a history of thrombotic events, may have also prevented a correlation [1]. Hemorrhaging has been speculated to be due to endothelial cell dysfunction due to inhibition of VEGF signaling, inhibition of tissue factor secretion, and necrosis of vascularly infiltrated tumor tissue following treatment with VEGFR-TKIs such as axitinib, pazopanib, sorafenib, and sunitinib $[10,11]$. No correlation was found for hemorrhage terms (excluding laboratory terms). On the other hand, in hemorrhagic central nervous system vascular conditions, there was no significant correlation. However, there was a trend toward a correlation, and a relatively strong correlation was obtained. Due to the wide scope of SMQ coverage, there may be a mix of reports of non-TKI-related hemorrhage terms (excluding laboratory terms) and hemorrhagic central nervous system vascular conditions, which may not have been correlated in the present results.

Among VEGFR2-related adverse events, the mechanism for the development of hypothyroidism is unclear [1]. However, a relationship between VEGFR-TKIs and hypothyroidism has been reported. It has been speculated that its mechanism of development includes inhibition of iodine uptake by thyroid follicular cells, decreased thyroid blood flow due to VEGFR suppression, inhibition of monocarboxylate transporter 8 (which is one of the thyroid hormone membrane 
Table 4 Sensitivity analysis

\begin{tabular}{|c|c|c|c|c|c|c|}
\hline \multirow[t]{2}{*}{ SMQ } & \multicolumn{3}{|c|}{ Sensitivity analysis 1} & \multicolumn{3}{|c|}{ Sensitivity analysis 2} \\
\hline & $r$ & $p$ & $\mathrm{SL}^{\mathrm{a}}$ & $r$ & $p$ & $\mathrm{SL}^{\mathrm{a}}$ \\
\hline Hypertension & $0.64^{*}$ & 0.0057 & 0.0125 & 0.60 & 0.0412 & 0.0063 \\
\hline Proteinuria & $0.58^{*}$ & 0.0152 & 0.0188 & 0.50 & 0.0985 & 0.0188 \\
\hline Embolic and thrombotic events, arterial & 0.18 & 0.4965 & 0.0438 & 0.20 & 0.5282 & 0.0438 \\
\hline Hemorrhage terms (excl laboratory terms) & 0.39 & 0.1226 & 0.0375 & 0.12 & 0.7007 & 0.0500 \\
\hline Hemorrhagic CNS vascular conditions & 0.53 & 0.0280 & 0.0250 & 0.36 & 0.2478 & 0.0375 \\
\hline Gastrointestinal perforation & $0.75^{*}$ & 0.0005 & 0.0063 & 0.52 & 0.0818 & 0.0125 \\
\hline Severe cutaneous adverse reactions & 0.02 & 0.9544 & 0.0500 & 0.41 & 0.1879 & 0.0250 \\
\hline Noninfectious diarrhea & 0.48 & 0.0538 & 0.0312 & 0.39 & 0.2117 & 0.0312 \\
\hline
\end{tabular}

SMQ Standardised MedDRA Queries, CNS central nervous system

$\mathrm{r}>0.4$ : bolded and italicized

*Significant difference

${ }^{a}$ Significance level by false discovery rate

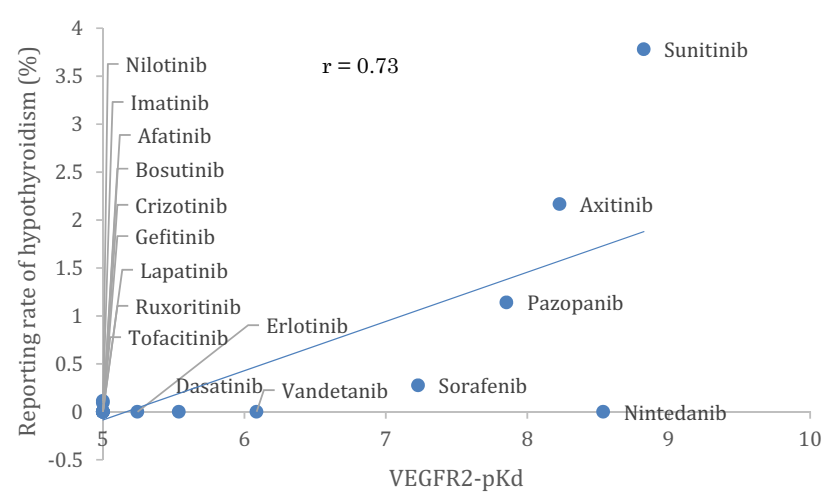

Fig. 1 Scatter plots showing the relationship between the reporting rate of hypothyroidism and protein kinase inhibitors' affinity (pKd) for protein kinase vascular endothelial growth factor receptor (VEGFR) 2

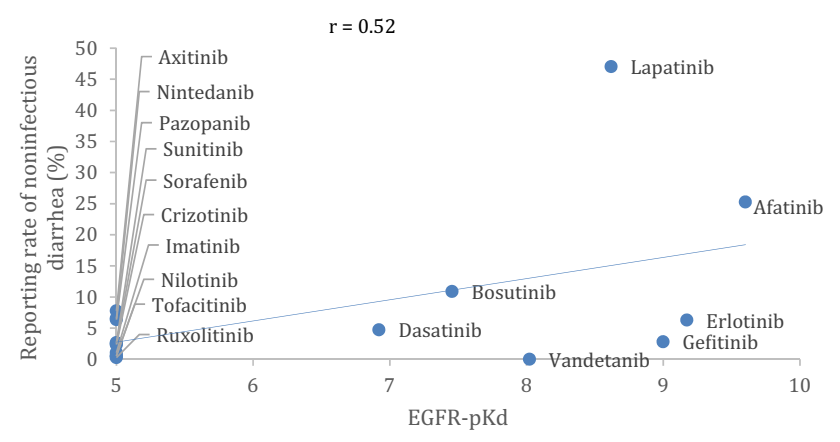

Fig. 2 Scatter plots showing the relationship between the reporting rate of noninfectious diarrhea and protein kinase inhibitors' affinity (pKd) for protein kinase epidermal growth factor receptor (EGFR) transport proteins), induction of destructive thyroiditis, and suppression of thyroid hormone feedback on pituitary TSH production and secretion [16-20]. The kinase adverse event correlation map also suggests that we have found a relationship between VEGFR2 and hypothyroidism. This suggests that a higher affinity for VEGFR2 may increase the risk of developing hypothyroidism. Hypothyroidism often goes unnoticed until subjective symptoms appear, and if we do not suspect it, the test is not performed, so TKIs with an affinity for VEGFR2 must be monitored in clinical practice. Noteworthy here is nintedanib. Nintedanib has the second highest affinity for VEGFR2 after sunitinib (Fig. 1). Nonetheless, the reporting rate of hypothyroidism for nintedanib is $0 \%$ (Table 2). Hypothyroidism has been described in foreign package inserts for nintedanib. In contrast, in Japan hypothyroidism is not listed there, so it may be overlooked in clinical practice. Therefore, as with axitinib, pazopanib, sorafenib, and sunitinib, monitoring in clinical practice is considered necessary.

EGFR-TKIs, which target EGFR, are considered one of the standard treatments for patients with mutation-positive non-small-cell lung cancer. EGFR is expressed in epithelial cells throughout the gastrointestinal tract, and diarrhea caused by EGFR-TKIs is one common adverse event. The mechanism of diarrhea caused by EGFR-TKIs has been attributed to a combination of factors, including changes in gastrointestinal motility and intestinal microbiota, and there are many unanswered questions about it [21]. One hypothesis is that epidermal growth factor acts on $\mathrm{Ca}^{2+}$-dependent $\mathrm{Cl}^{-}$channels in intestinal epithelial cells to inhibit intestinal $\mathrm{Cl}^{-}$secretion, as seen in an in vitro study [2], and this may be the mechanism by which EGFR-TKIs increase $\mathrm{Cl}^{-}$secretion into the intestinal tract, which in turn increases water secretion and leads to diarrhea. In our study, there was also a relationship between EGFR and diarrhea and dehydration, 
supporting that the high affinity of EGFR is a factor in diarrhea and associated dehydration (see OSM).

EGFR-TKIs, bosutinib, lapatinib, and vandetanib are listed in the package insert as having serious side effects and have been warned about in clinical practice. However, with dasatinib, diarrhea is not listed in the serious side-effect section in the package insert and the warning about diarrhea is weaker than with other TKIs that have an affinity for EGFR, suggesting that clinical measures against it may not be sufficient. Diarrhea can cause anxiety, fatigue, and sleep disturbance, preventing patients from going out and potentially affecting their social life [22], all of which can have a significant impact on the patient's QOL and lead to nonadherence. Non-adherence in patients with CML is known to affect patients' life expectancy [23], and in CML, where long-term survival is observed, a reduction in patients' QOL due to diarrhea may lead to non-adherence, which in turn may lead to a shorter life expectancy. Therefore, the reduction in QOL of patients due to diarrhea caused by dasatinib needs to be avoided and should be addressed as soon as possible in clinical practice.

The JADER database and other adverse event spontaneous reporting databases have several problems, including under-reporting and reporting bias that is susceptible to market trends [24]. Therefore, great care must be taken in interpreting the results. The World Health Organization (WHO) defines a signal as "reported information on a possible causal relationship between an adverse event and a drug, the relationship being unknown or incompletely documented previously" [25]. CIOMS states that statistical analysis of the adverse event spontaneous reporting database is not a substitute for careful and appropriate clinical judgment, and shows the need for and importance of adverse event assessment based on the spontaneous reporting system [7]. In this study, the reporting rate was used as a surrogate indicator of the incidence of adverse events. However, as time passes after a drug's approval, less serious reactions may not be reported and the reporting rate may become artificially low. In other words, it is necessary to recognize that it is like a snapshot of a point in time. However, this is a limitation of using spontaneous reports because the ROR and the proportional reporting ratio are still the reporting ratios at that point in time. In addition, there may be confounding factors such as indications, underlying diseases, and concomitant medications. Furthermore, the doses of drugs approved in Japan are often lower than in other countries, and there are ethnic differences in drug responses. In other words, signal detection using an adverse event spontaneous report database is a stage of risk hypothesis, and it is necessary to evaluate them further.

Therefore, the association between kinase affinity and adverse events visualized in this study cannot be considered causal. Of the eight adverse events for which correlations were tested, no correlation was found for embolic and thrombotic events, arterial, hemorrhage terms (excluding laboratory terms), and hemorrhagic central nervous system vascular conditions, suggesting that caution should be exercised in interpreting results when the pathogenic mechanism can be explained by a single kinase affinity or when confounding factors cannot be ruled out. However, in the development of molecularly targeted therapies, there is a need for more attention to be paid to the mechanism of action.

The rate of development of molecular targeted therapy has been remarkable, and because of its great efficacy, it is widely used in clinical practice and its indications are expanded more frequently than other drugs. In such an environment, we have encountered cases in which patients are forced to discontinue treatment due to unexpected adverse events and do not receive the full benefit of molecularly targeted therapies. The results of this study are correlational and hypothesis-generating with exploratory results, but they are an important alert until these cause-and-effect relationships can be clarified in a more validated manner. Knowing these results may help to visualize the risk of potential adverse events due to off-target effects, which are difficult to see in actual clinical practice, and may lead to aiding management in actual clinical practice.

We included 17 TKIs for which Kd were obtained in the report of Davis et al. as basic data [3]. However, because the number of TKIs available today is increasing, there are some TKIs that have not been studied. In addition, the data are based on kinase affinity only, and do not take into account plasma concentration. Therefore, it is desirable to have more basic data for the monitoring of adverse events in clinical practice. However, we were able to perform a comprehensive study of the adverse events of TKIs, which may be difficult to obtain in clinical practice. By calculating the correlation coefficient between kinase affinity and adverse events, we not only visualized the relationship between kinase affinity and adverse events, but also identified the possibility of overlooking adverse events caused by nintedanib, information that could be utilized in the management of TKIs in clinical practice. Even though there are multiple causes of diarrhea, our results can help to determine how bosutinib and dasatinib can be used in clinical settings to prevent diarrhea, even though the mechanism causing diarrhea has remained unclear. These results suggest that appropriate monitoring may prevent unnecessary chemotherapy interruptions and improve the life expectancy and quality of life of cancer patients, as well as preventing more serious adverse events in patients receiving nintedanib, bosutinib, and dasatinib. 


\section{Conclusions}

Our comprehensive analysis of the kinase affinities of TKIs in this study, which was based on the basic data of kinase affinity of TKIs and the clinical data of the reporting rates, suggested that our comprehensive analysis method is useful for generating hypotheses about possible causal relationships between pharmacological effects and adverse events. Our findings suggest that this comprehensive method of analyzing kinase affinity and adverse events is useful in finding adverse events that are difficult to notice.

Supplementary Information The online version contains supplementary material available at https://doi.org/10.1007/s40801-021-00235-w.

Acknowledgements Not applicable.

\section{Declarations}

Funding Not applicable.

Conflicts of interest TM, TS, KT, TU, NG, and FO have no conflicts of interest directly relevant to the content of this study.

Ethics approval Not applicable.

Availability of data and material (data transparency) The data are available from the corresponding author on reasonable request.

Code availability (software application or custom code) Not applicable.

Authors' contributions TM, FO, KT, NG, and TU were involved in the conception and design of the study; TM and TS contributed to the data extraction; TM and TS conducted the analyses; and all authors contributed to interpreting the data and reviewed and edited the manuscript. All authors attest they meet the International Committee of Medical Journal Editors criteria for authorship.

Open Access This article is licensed under a Creative Commons Attribution-NonCommercial 4.0 International License, which permits any non-commercial use, sharing, adaptation, distribution and reproduction in any medium or format, as long as you give appropriate credit to the original author(s) and the source, provide a link to the Creative Commons licence, and indicate if changes were made. The images or other third party material in this article are included in the article's Creative Commons licence, unless indicated otherwise in a credit line to the material. If material is not included in the article's Creative Commons licence and your intended use is not permitted by statutory regulation or exceeds the permitted use, you will need to obtain permission directly from the copyright holder. To view a copy of this licence, visit http://creativecommons.org/licenses/by-nc/4.0/.

\section{References}

1. Chen HX, Cleck JN. Adverse effects of anticancer agents that target the VEGF pathway. Nat Rev Clin Oncol. 2009;6:465-77.

2. Uribe JM, Gelbmann CM, Traynor-Kaplan AE, Barrett KE. Epidermal growth factor inhibits $\mathrm{Ca}(2+)$-dependent $\mathrm{Cl}$ - transport in
T84 human colonic epithelial cells. Am J Physiol. 1996;271(3 Pt 1):C914-22.

3. Davis M, Hunt J, Herrgard S, Ciceri P, Wodicka LM, Pallares G, et al. Comprehensive analysis of kinase inhibitor selectivity. Nat Biotechnol. 2011;29:1046-51.

4. Mahé J, de Campaigno EP, Chené AL, Montastruc JL, Despas F, Jolliet P. Pleural adverse drugs reactions and protein kinase inhibitors: identification of suspicious targets by disproportionality analysis from VigiBase. Br J Clin Pharmacol. 2018;84(10):2373-83.

5. Yamamoto N, Honma M, Suzuki H. Off-target serine/threonine kinase 10 inhibition by erlotinib enhances lymphocytic activity leading to severe skin disorders. Mol Pharmacol. 2011;80(3):466-75.

6. Siafis Spyridon, Papazisis Georgios. Detecting a potential safety signal of antidepressants and type 2 diabetes: a pharmacovigilance-pharmacodynamic study. Br J Clin Pharmacol. 2018;84(10):2405-14.

7. CIOMS Working Group VIII. Practical aspects of signal detection in pharmacovigilance. CIOMS Geneva; 2010.

8. Agarwal M, Thareja N, Benjamin M, Akhondi A, Mitchell GD. Tyrosine kinase inhibitor-induced hypertension. Curr Oncol Rep. 2018;20(8):65.

9. Eremina V, Jefferson JA, Kowalewska J, Hochster H, Haas M, Weisstuch J, et al. VEGF inhibition and renal thrombotic microangiopathy. N Engl J Med. 2008;358(11):1129-36.

10. Verheul HMW, Pinedo HM. Possible molecular mechanisms involved in the toxicity of angiogenesis inhibition. Nat Rev Cancer. 2007;7(6):475-85.

11. Kamba T, McDonald DM. Mechanisms of adverse effects of antiVEGF therapy for cancer. Br J Cancer. 2007;96(12):1788-95.

12. Qi WX, Sun YJ, Tang LN, Shen Z, Yao Y. Risk of gastrointestinal perforation in cancer patients treated with vascular endothelial growth factor receptor tyrosine kinase inhibitors: a systematic review and meta-analysis. Crit Rev Oncol Hematol. 2014;89(3):394-403.

13. Shah RR. Anti-angiogenic tyrosine kinase inhibitors and reversible posterior leukoencephalopathy syndrome: could hypomagnesaemia be the trigger? Drug Saf. 2017;40(5):373-86.

14. Theonie Anastassiadis, Sean W Deacon, Karthik Devarajan, Haiching Ma, Jeffrey R Peterson. Comprehensive assay of kinase catalytic activity reveals features of kinase inhibitor selectivity. Nat Biotechnol. 2011;30;29(11):1039-45.

15. Valent P, Hadzijusufovic E, Hoermann G, Füreder W, Schernthaner GH, Sperr WR, et al. Risk factors and mechanisms contributing to TKI-induced vascular events in patients with CML. Leuk Res. 2017;59:47-54.

16. Mannavola D, Coco P, Vannucchi G, Bertuelli R, Carletto M, Casali PG, et al. A novel tyrosine-kinase selective inhibitor, sunitinib, induces transient hypothyroidism by blocking iodine uptake. J Clin Endocrinol Metab. 2007;92(9):3531-4.

17. Kamba T, Tam BY, Hashizume H, Haskell A, Sennino B, Mancuso MR, et al. VEGF-dependent plasticity of fenestrated capillaries in the normal adult microvasculature. Am J Physiol Heart Circ Physiol. 2006;290(2):H560-76.

18. Braun D, Kim TD, le Coutre P, Köhrle J, Hershman JM, Schweizer U. Tyrosine kinase inhibitors noncompetitively inhibit MCT8-mediated iodothyronine transport. J Clin Endocrinol Metab. 2012;97(1):E100-5.

19. Wong E, Rosen LS, Mulay M, Vanvugt A, Dinolfo M, Tomoda $\mathrm{C}$, et al. Sunitinib induces hypothyroidism in advanced cancer patients and may inhibit thyroid peroxidase activity. Thyroid. 2007;17(4):351-5.

20. de Groot JW, Links TP, van der Graaf WT. Tyrosine kinase inhibitors causing hypothyroidism in a patient on levothyroxine. Ann Oncol. 2006;17(11):1719-20. 
21. Yang JC, Reguart N, Barinoff J, Köhler J, Uttenreuther-Fischer M, Stammberger U, et al. Diarrhea associated with afatinib: an oral ErbB family blocker. Expert Rev Anticancer Ther. 2013;13(6):729-36.

22. Hirsh V, Blais N, Burkes R, Verma S, Croitoru K. Management of diarrhea induced by epidermal growth factor receptor tyrosine kinase inhibitors. Curr Oncol. 2014;21(6):329-36.

23. Marin D, Bazeos A, Mahon FX, Eliasson L, Milojkovic D, Bua $\mathrm{M}$, et al. Adherence is the critical factor for achieving molecular responses in patients with chronic myeloid leukemia who achieve complete cytogenetic responses on imatinib. J Clin Oncol. 2010;28(14):2381-8.
24. Poluzzi E, Raschi E, Moretti U, Ponti FD. Drug-induced torsades de pointes: data mining of the public version of the FDA Adverse Event Reporting System (AERS). Pharmacoepidemiol Drug Saf. 2009;18(6):512-8.

25. Edwards IR, Lindquist M, Wiholm BE, Napke E. Quality criteria for early signals of possible adverse drug reactions. Lancet. 1990;336(8708):156-8. 